\title{
A pragmatic, randomized, controlled study evaluating the impact of access to smoking cessation pharmacotherapy coverage on the proportion of successful quitters in a Canadian population of smokers motivated to quit (ACCESSATION)
}

Peter Selby ${ }^{1,2,3^{*}+}$, Gerald Brosky ${ }^{4 \dagger}$, Paul Oh ${ }^{5 \dagger}$, Vincent Raymond ${ }^{6 \dagger}$, Carmen Arteaga ${ }^{7 \dagger}$ and Suzanne Ranger ${ }^{8 \dagger}$

\begin{abstract}
Background: Many smokers find the cost of smoking cessation medications a barrier. Financial coverage for these medications increases utilization of pharmacotherapies. This study assesses whether financial coverage increases the proportion of successful quitters.

Methods: A pragmatic, open-label, randomized, controlled trial was conducted in 58 Canadian sites between March 2009 and September 2010. Smokers ( $\geq 10$ cigarettes/day) without insurance coverage who were motivated to quit within 14 days were randomized (1:1) in a blinded manner to receive either full coverage eligibility for 26 weeks or no coverage. Pharmacotherapies covered were varenicline, bupropion, or nicotine patches/gum. Investigators/subjects were unblinded to study group assignment after randomization and prior to choosing a smoking cessation method(s). All subjects received brief smoking cessation counseling. The primary outcome measure was self-reported 7-day point prevalence of abstinence (PPA) at week 26.

Results: Of the 1380 randomized subjects (coverage, 696; no coverage, 684), 682 (98.0\%) and 435 (63.6\%), respectively, were dispensed at least one smoking cessation medication dose. The 7-day PPA at week 26 was higher in the full coverage versus no coverage group: $20.8 \%(n=145)$ and $13.9 \%(n=95)$, respectively; odds ratio $(\mathrm{OR})=1.64,95 \%$ confidence interval (Cl) 1.23-2.18; $p=0.001$. Urine cotinine-confirmed 7-day PPA at week 26 was $15.7 \%(n=109)$ and $10.1 \%(n=69)$, respectively; $O R=1.68,95 \% \mathrm{Cl} 1.21-2.33 ; p=0.002$. After pharmacotherapy, coverage eligibility was withdrawn from the full coverage group, continuous abstinence between weeks 26 and 52 was $6.6 \%$ ( $n=46$ ) and $5.6 \%(n=38)$, in the full coverage and no coverage groups, respectively; $\mathrm{OR}=1.19,95 \% \mathrm{Cl} 0.76-1.87 ; p=0.439$. (Continued on next page)
\end{abstract}

\footnotetext{
*Correspondence: peter.selby@camh.ca

${ }^{\dagger}$ Equal contributors

${ }^{1}$ Addictions Division, Centre for Addiction and Mental Health, Toronto,

Ontario, Canada

${ }^{2}$ Departments of Family and Community Medicine and Psychiatry and the Dalla Lana School of Public Health, University of Toronto, Toronto, Ontario,

Canada

Full list of author information is available at the end of the article
}

C Biomed Central (c) 2014 Selby et al.; licensee BioMed Central Ltd. This is an Open Access article distributed under the terms of the Creative Commons Attribution License (http://creativecommons.org/licenses/by/2.0), which permits unrestricted use, distribution, and reproduction in any medium, provided the original work is properly credited. The Creative Commons Public Domain Dedication waiver (http://creativecommons.org/publicdomain/zero/1.0/) applies to the data made available in this article, unless otherwise stated. 
(Continued from previous page)

Conclusions: In this study, the adoption of a smoking cessation medication coverage drug policy was an effective intervention to improve 26-week quit rates in Canada. The advantages were lost once coverage was discontinued. Further study is required on the duration of coverage to prevent relapse to smoking. (clinicaltrials.gov identifier: NCT00818207; the study was sponsored by Pfizer Inc.).

Keywords: Bupropion, Clinical trial, Medications, Nicotine, Pharmacotherapy coverage, Policy, Pragmatic, Reimbursement, Smoking cessation, Varenicline

\section{Background}

Tobacco addiction is associated with one in eight deaths worldwide [1]. The efficacy of all smoking cessation medications (pharmacotherapy) i.e., varenicline, bupropion, and nicotine replacement therapies (NRTs; patch, gum, lozenge, inhaler, and nasal spray) is well established [2-6]. Nicotine replacement therapies (odds ratio $[\mathrm{OR}]=1.89$, credible interval [CrI] 95\% 1.63-2.18), bupropion ( $\mathrm{OR}=1.95$, CrI 95\% 1.58-2.41), and varenicline $(\mathrm{OR}=2.78$, CrI 95\% 2.17-3.57) all increase the odds of 12-month abstinence compared with placebo [5]. Although there are many reasons why smokers do not access pharmacotherapy, lack of insurance coverage remains a significant barrier [7]. Pharmacotherapy coverage may increase the number of quit attempts, the likelihood of pharmacotherapy utilization and adherence, and ultimately, smoking cessation.

Despite pharmacotherapies being among the most costeffective and highly advocated interventions for coverage [8], funders have been slow to invest. Systematic reviews concluded that full coverage (versus no coverage) of pharmacotherapies could increase quit rates with low attributable incremental cost $[5,9]$. However, the included trials typically excluded real-world smokers who often have psychiatric and medical comorbidity, thereby limiting generalizability [10]. The few published pragmatic studies have several methodological problems such as not being randomized controlled trials (RCTs) and not including all available pharmacotherapies [9].

Based on the 10 dimensions of the PRECIS Tool to evaluate clinical trial design $[11,12]$, this mostly pragmatic open-label RCT evaluated the effectiveness of pharmacotherapy coverage versus no coverage for 26 continuous weeks in increasing smoking cessation in ambulatory care settings in motivated adult smokers in Canada.

\section{Methods}

\section{Study design and participants}

This open-label RCT was conducted in 58 different sites across Canada (excepting Quebec, Alberta, and New Brunswick). Smokers were recruited by study site referrals and advertisement. Potential subjects were screened by a call centre to assess initial eligibility and drug insurance coverage. The investigator then confirmed eligibility (see Additional file 1).
Participants were adults (18-75 years) who smoked $\geq 10$ cigarettes per day, were willing to set a quit date within 14 days following screening/randomization, had no period of abstinence $>3$ months in the past year, and had not attempted to quit smoking in the 30-day period before screening. Exclusion criteria were: unknown insurance status, access to unused pharmacotherapy or prescription, use of tobacco products other than cigarettes within the past month, and life-threatening illness (e.g., known or suspected cancer or other disease with a life expectancy of $<1$ year). Medical and psychiatric conditions were not exclusion criteria per se.

The study was conducted in compliance with the ethical principles originating in or derived from the Declaration of Helsinki and in compliance with all International Conference on Harmonization Good Clinical Practice Guidelines. Written informed consent for participation in the study was obtained from all participants. The final protocol, any amendments, and informed consent documentation were reviewed and approved by the Canadian Institutional Review Board (IRB) (IRB Services, Aurora, ON, Canada), or by local Canadian Ethics Committees (University of British Columbia Clinical Research Ethics Board, Vancouver, BC; Vancouver Island Health Authority Clinical Research Ethics Board, Victoria, BC; University of Manitoba Research Ethics, Winnipeg, MB; Winnipeg Clinic Ethics Committee, Winnipeg, MB; Research Ethics Board St. Joseph's Healthcare Hamilton, Hamilton, ON; University of Western Ontario Office of Research Ethics, London, $\mathrm{ON}$ ).

\section{Randomization and interventions}

Eligible participants were first counseled about smoking cessation and then randomly and blindly assigned (1:1; centrally to prevent gaming) at the baseline visit to receive either full coverage for 26 weeks or no coverage. Randomization was performed by the investigators using blinded lots of computer-generated randomization codes from the study biostatistician. Randomization codes were mapped to SmartPayment ${ }^{\mathrm{TM}}$ cards (drug reimbursement cards), with a distinctive color linked to the study arm to which the subject was randomized. The SmartPayment ${ }^{\mathrm{mm}}$ cards were enclosed in sealed envelopes with the randomization codes printed on the envelopes. After 
unblinding, the investigator and participant agreed on the pharmacological and/or non-pharmacological smoking cessation method(s) to be used. Eligible pharmacotherapies were: varenicline; bupropion; and NRT patch and gum, prescribed according to the most recent version of the respective product monograph or equivalent (see Additional file 2). A prescription had to be issued by a physician for these pharmacotherapies to be reimbursed; NRTs obtained without a prescription were not eligible for coverage, but were considered medication for statistical analysis. Participants (irrespective of allocated group) were not obliged to use a pharmacotherapy in their quit attempt. A target quit date was set within 14 days of randomization. The use of a SmartPayment ${ }^{\text {tw }}$ card permitted access to either full coverage of prescribed pharmacotherapy for 26 weeks (full coverage) or a reimbursement of $\$ 5.00$ per pharmacy dispensation (no coverage). This facilitated tracking of prescriptions dispensed in both groups and provided an incentive for subjects in the no coverage group to present their card at each pharmacy visit. Participants received compensation for reasonable expenses for transportation or parking for study appointments, if needed.

\section{Assessments and follow-up}

Patient assessment, treatment recommendations, and prescriptions, as needed, were performed by physicians. Other study-related tasks were performed by trained personnel. Participants were screened at the baseline visit for demographic information, type of drug plan insurance, smoking history, Fagerström Test for Nicotine Dependence (FTND) score [13,14], comorbid medical/ psychiatric history including the Columbia Suicide Severity Rating Scale (C-SSRS [15]), and concomitant medications. Brief (10-minute) smoking cessation counseling was provided to all subjects at the screening visit and at each contact, per each site's practice. Prior to study start, all participating sites were provided brief training in current clinical recommendations/guidelines [16]. Participants made four clinic visits (weeks 1, 26, 39, and 52), and received six telephone calls (weeks 2, 13, $30,34,44$, and 48), during which smoking status, quit attempt(s)/methods, FTND score, and abstinence status (via self-report at clinic visits) were collected. Confirmatory urinary cotinine measurements were collected, but investigators and patients were blinded to the results to mimic real-world practice (where this is not widely used). Patients were not aware that physicians were blinded to the results.

Safety evaluations included adverse event (AE) monitoring, physical examinations, and C-SSRS [15]. All observed or volunteered AEs, regardless of smoking cessation method or suspected causal relationship, were to be reported. The investigator was required to pursue information to both determine the outcome of the $\mathrm{AE}$ and assess whether it met criteria for a serious AE. Events were recorded up to and including 28 days after the last administration of one of the smoking cessation methods or week 26 (whichever was longer).

\section{Effectiveness evaluations}

The primary endpoint was self-reported 7-day point prevalence of abstinence (PPA) at week 26 (end of coverage eligibility), defined as abstinence from smoking (not a single puff) for at least the preceding 7 days [17]. This pragmatic endpoint was chosen because in real-world settings there is no evidence of significant difference between estimates of smoking prevalence based on self-report versus urinary cotinine measure [18]. Whereas the primary endpoint was more pragmatic, the key secondary endpoints (self-reported 7-day PPA at week 26 and continuous abstinence rates [CAR] at weeks $26-52$, confirmed by urine cotinine) were more explanatory. Other secondary endpoints were self-reported 7-day PPA at weeks 2 and 13, self-reported CAR (weeks 26-39), total number of quit attempts from randomization through week 26 , and use of smoking cessation pharmacotherapies during quit attempts.

\section{Statistical analysis}

Based on previous clinical studies of varenicline $[19,20]$, market research, and relevant literature [21-24], quit rates in the no coverage versus full coverage group were assumed to be $15 \%$ versus $21 \%$, respectively. A sample size of at least 676 participants per group was estimated to provide $80 \%$ power to detect a conservative difference of $6 \%$ between groups. Some consider a $2 \%$ difference clinically significant because of the estimated 50\% risk reduction in smoking-related mortality associated with cessation [25]. Outcome measures were assessed using an intent-to-treat (ITT) analysis that included all randomized participants. Individuals who discontinued the study or were lost to follow-up were counted as smokers from the time of discontinuation. For calculation of CAR, participants who reported no smoking or no use of nicotine since the last contact and had a negative urine cotinine test were counted as non-smokers. Participants with missing urine cotinine test results were considered smokers.

Endpoints were analyzed using logistic regression models that included randomized group effect (full coverage versus non-coverage) as the independent variable and investigation centre as a covariate. In addition, the interaction between randomized group and centre was tested by expanding the logistic regression model to add the interaction term. In testing for the primary and key secondary endpoints, the Type-I overall error rate of 0.05 was preserved by using statistical methods that adjusted for multiple tests. 
Safety data were summarized for all randomized subjects. The incidence of AEs reported during the study was summarized by randomized arm (coverage; non-coverage) and pharmacotherapy (users; non-users) for descriptive rather than comparative purposes.

\section{Results}

\section{Baseline characteristics}

Eligible participants were recruited from March 3, 2009 to September 20, 2010. Participants attended clinic visits at the time of randomization and at regular intervals for 52 weeks. Using postal codes as a proxy, participating sites were identified as being both in urban (54) and rural (4) locations. Participants were recruited by eight specialists $(n=115$; $8.3 \%)$ and 50 generalists $(n=1265 ; 91.7 \%)$ and were referred from clinical practice $(n=1289 ; 93.4 \%)$ or advertisement $(n=91 ; 6.6 \%)$. Participant disposition is summarized in Figure 1. Baseline demographics, smoking history, and level of nicotine dependence were well balanced (Table 1). Interactions for randomization group by centre were not statistically significant $(p>0.4)$, supporting the consistency of using combined results from different centres. The primary analysis was ITT and involved all patients who were randomly assigned (696 subjects in the full coverage group and 684 subjects in the no coverage group). Participants were analysed by original group assignment.

\section{Effectiveness of coverage of pharmacotherapy}

Significantly more participants in the full coverage versus no coverage group achieved the primary endpoint of 7-day PPA at week 26 (20.8\% versus $13.9 \%$, respectively; odds ratio $(\mathrm{OR})=1.64,95 \%$ confidence interval $(\mathrm{CI})$ $1.23-2.18 ; p=0.001$; Table 2). Significant differences in 7 -day PPA between the full coverage and no coverage groups were observed at week $2(18.8 \%$ versus $13.3 \%$; $p=0.003)$ and at week $13(34.1 \%$ versus $23.7 \% ; p<0.001)$. The difference remained significant for urine cotinineconfirmed 7-day PPA at week $26(15.7 \%$ versus $10.1 \%$, respectively; $\mathrm{OR}=1.68,95 \% \mathrm{CI} 1.21-2.33 ; p=0.002$; Table 2). After withdrawal of coverage eligibility, there was no significant difference in CAR from weeks 26 to 52 between the two groups (week $52, n=6.6 \%$ versus $n=5.6 \%$, respectively; OR $=1.19,95 \%$ CI $0.76-1.87$; $p=0.439$; see Additional file 3). Similar results were noted at week $39(7.8 \%$ versus $6.3 \%$, respectively; $\mathrm{OR}=1.25$, 95\% CI 0.82-1.90; $p=0.298$ ). However, 7-day PPA data were not collected after 26 weeks and the CAR at weeks 26-52 should not be directly compared with 7-day PPA data. A post-hoc analysis showed that pharmacotherapy utilization peaked at week $2(\sim 90 \%$ in the full coverage group), after which it steadily decreased for the remainder of the intervention phase, reaching approximately $20 \%$ at week 26 (see Additional file 4).

During the 26-week coverage eligibility period, a higher proportion of participants in the full coverage versus no coverage group made at least one quit attempt $(86.9 \%$ versus $70.0 \%$, respectively; $p<0.001$ ), but the number of quit attempts were not statistically different across groups (mean 3.1 versus 2.6, respectively; $p=0.103$ ), and more subjects were dispensed at least one pharmacotherapy

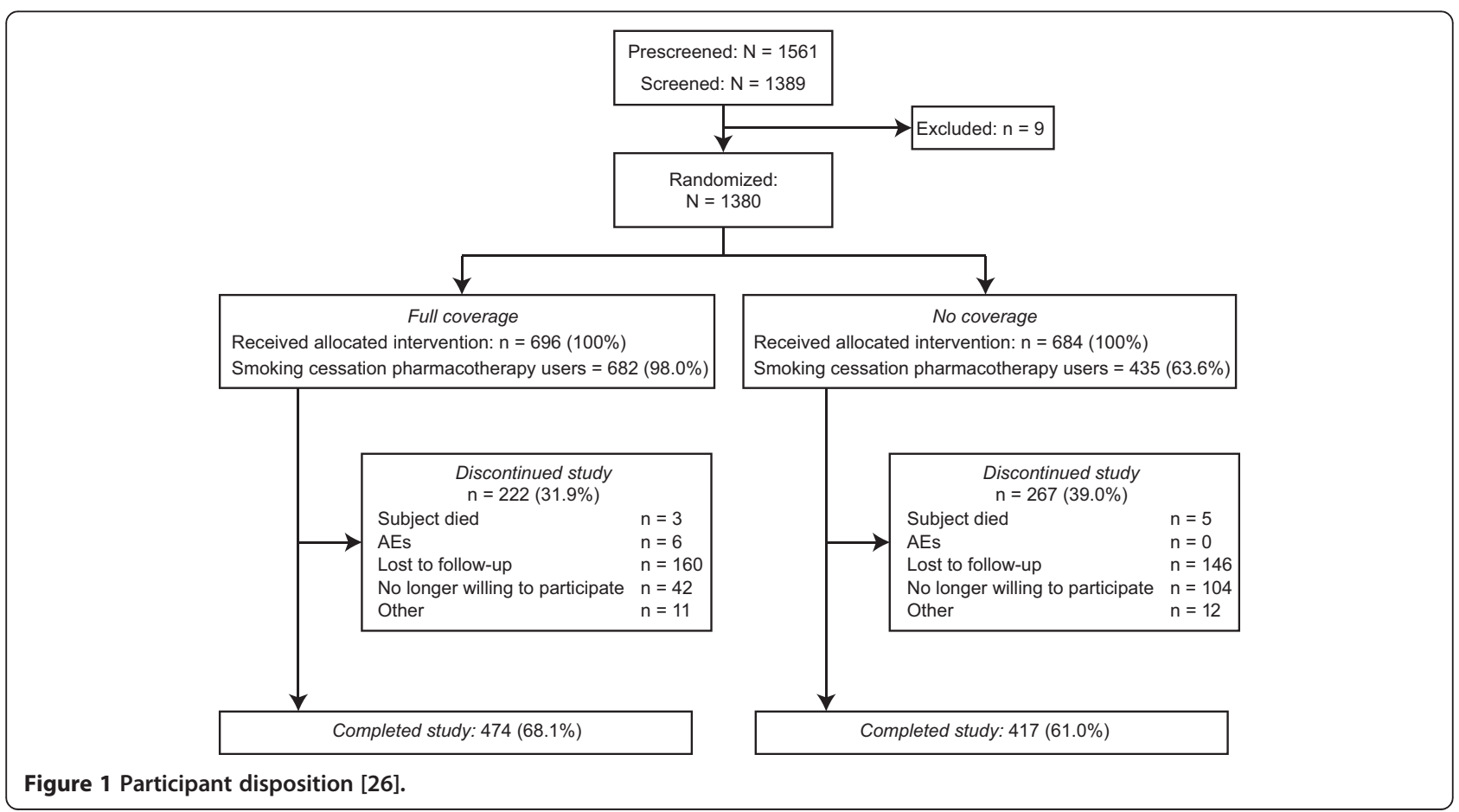


Table 1 Baseline characteristics of study participants

\begin{tabular}{|c|c|c|}
\hline & $\begin{array}{l}\text { Full coverage } \\
\qquad(n=696)\end{array}$ & $\begin{array}{l}\text { No coverage } \\
(n=684)\end{array}$ \\
\hline \multicolumn{3}{|l|}{ Demographic characteristics } \\
\hline Age, mean, years (SD) & $46.5(12.3)$ & $46.7(12.3)$ \\
\hline \multicolumn{3}{|l|}{ Gender, n (\%) } \\
\hline Male & $354(50.9)$ & $337(49.3)$ \\
\hline Female & $342(49.1)$ & $347(50.7)$ \\
\hline \multicolumn{3}{|l|}{ Race, $n(\%)$} \\
\hline White & $664(95.4)$ & $660(96.5)$ \\
\hline Black & $11(1.6)$ & $6(0.9)$ \\
\hline Asian & $15(2.2)$ & $13(1.9)$ \\
\hline Other & $6(0.9)$ & $5(0.7)$ \\
\hline \multicolumn{3}{|l|}{ Educational level, years, n (\%) } \\
\hline$<10$ & $46(6.6)$ & $57(8.3)$ \\
\hline $10-12$ & $321(46.1)$ & $305(44.6)$ \\
\hline $13-16$ & $273(39.2)$ & $257(37.6)$ \\
\hline$>16$ & $56(8.0)$ & $61(8.9)$ \\
\hline \multicolumn{3}{|l|}{ Employment status, $n(\%)$} \\
\hline Full-time employed & $298(42.8)$ & $297(43.4)$ \\
\hline Part-time employed & $79(11.4)$ & $77(11.3)$ \\
\hline Not employed & $319(45.8)$ & $308(45.0)$ \\
\hline \multicolumn{3}{|l|}{ Insurance type, n (\%) } \\
\hline Private insurance & $51(7.3)$ & $53(7.7)$ \\
\hline $\begin{array}{l}\text { Government-provided } \\
\text { insurance }\end{array}$ & $143(20.5)$ & $137(20.0)$ \\
\hline No drug insurance & $484(69.5)$ & $479(70.0)$ \\
\hline Other type of insurance & $18(2.6)$ & $14(2.0)$ \\
\hline \multicolumn{3}{|l|}{ Household income, $n$ (\%) } \\
\hline$<\$ 15,000$ CAD & $58(8.3)$ & $92(9.1)$ \\
\hline$\$ 15,000-<\$ 25,000 C A D$ & $260(37.4)$ & $246(36.0)$ \\
\hline$\$ 25,000-<\$ 50,000 C A D$ & $203(29.2)$ & $218(31.9)$ \\
\hline$\$ 50,000-<\$ 75,000 C A D$ & $78(11.2)$ & $62(9.1)$ \\
\hline$\$ 75,000-<\$ 100,000 C A D$ & $32(4.6)$ & $31(4.5)$ \\
\hline$>\$ 100,000 \mathrm{CAD}$ & $22(3.2)$ & $17(2.5)$ \\
\hline $\begin{array}{l}\text { Body mass index }\left(\mathrm{kg} / \mathrm{m}^{2}\right) \text {, } \\
\text { mean (SD) }\end{array}$ & $28.1(6.3)$ & $28.3(6.5)$ \\
\hline \multicolumn{3}{|l|}{ Smoking history } \\
\hline $\begin{array}{l}\text { Age at onset of smoking, } \\
\text { years, mean (SD) }\end{array}$ & $15.5(4.5)$ & $15.5(4.0)$ \\
\hline Cigarette pack years, mean (SD) & $34.8(21.8)$ & $34.6(21.5)$ \\
\hline $\begin{array}{l}\text { Cigarettes/day (past month), } \\
\text { mean (range) }\end{array}$ & $22.2(9.5)$ & $21.5(8.3)$ \\
\hline FTND score, mean (SD)* & $5.9(2.1)$ & $5.9(2.1)$ \\
\hline \multicolumn{3}{|l|}{$\begin{array}{l}\text { Number of lifetime serious quit } \\
\text { attempts - any method, } n(\%)\end{array}$} \\
\hline 0 & $93(13.4)$ & $64(9.4)$ \\
\hline 1 & $163(23.4)$ & $166(24.3)$ \\
\hline
\end{tabular}

Table 1 Baseline characteristics of study participants

(Continued)

\begin{tabular}{lcc}
\hline 2 & $143(20.5)$ & $148(21.6)$ \\
$\geq 3$ & $297(42.7)$ & $306(44.7)$ \\
Comorbidities $^{\dagger}$ & & \\
COPD & $65(9.3)$ & $77(11.3)$ \\
Lung cancer & 0 & $2(0.3)$ \\
Coronary heart disease & $18(2.6)$ & $17(2.5)$ \\
Stroke & $1(0.1)$ & $2(0.3)$ \\
Asthma exacerbation & $76(10.9)$ & $66(9.6)$ \\
Past psychiatric disorder & $73(10.5)$ & $65(9.5)$ \\
Current psychiatric disorder & $230(33)$ & $213(31.1)$ \\
Current depression & $109(15.7)$ & $106(15.5)$ \\
Current anxiety/anxiety disorder & $66(9.5)$ & $61(9.0)$ \\
\hline
\end{tabular}

$\mathrm{CAD}=$ Canadian dollar; $\mathrm{COPD}=$ chronic obstructive pulmonary disease; FTND = Fagerström Test for Nicotine Dependence; $S D=$ standard deviation; $\mathrm{SES}=$ socioeconomic status.

* Scores range from 0 to 10 , with higher scores indicating greater nicotine dependence. ${ }^{\dagger}$ Comorbidities reported as "present" in subjects' medical history at the time of the screening/randomization visit.

(98.0\% versus $63.6 \%$, respectively; $p<0.001$ ). The median number of daily doses dispensed per participant was higher in the full versus no coverage group, except for bupropion (70 versus 42, respectively; $p<0.001$, for varenicline; 56 versus $18.5 ; p<0.001$, respectively, for NRTs; and 39 versus $34 ; p=0.454$, respectively, for bupropion).

In the full coverage group, varenicline was dispensed at least once for 558 participants (80.2\%), nicotine patches/gum for 201 participants (28.9\%), and bupropion for $60(8.6 \%)$ participants. The corresponding figures for the no coverage group were 297 (43.4\%), 126 (18.4\%), and 58 (8.5\%). In the full coverage group, $18.4 \%$ of participants used at least two distinct pharmacotherapies, whereas only $6.3 \%$ did so in the no coverage group. Non-pharmacological methods were used by $5.7 \%$ and $9.9 \%$, respectively.

\section{Adverse events}

Adverse events are listed in Table 3. Serious AEs considered by the investigator to be treatment-related were reported for two participants in the full coverage group (muscular weakness and hyperkalemia; both $n=1$ ) and two in the no coverage group (violence-related symptom and depression; both $n=1$ ). Results from the C-SSRS assessment were also similar between the groups and revealed a "yes" answer for suicidal ideation and/or behavior in $25(3.7 \%)$ and $11(2.5 \%)$ in the full coverage and no coverage groups, respectively. A suicidal attempt was reported in one subject who did not take pharmacotherapy. All-causality AEs reported for $\geq 5 \%$ of participants (randomized and used smoking cessation pharmacotherapy) in the full coverage and no coverage arms, respectively, 
Table 2 7-day point prevalence of abstinence from weeks 2 to 26 and complete abstinence rate from weeks 26 to 52 (intent-to-treat)

\begin{tabular}{|c|c|c|c|c|}
\hline & $\begin{array}{l}\text { Full coverage }(n=696) \text {, } \\
\text { proportion of patients, } \%\end{array}$ & $\begin{array}{l}\text { No coverage }(n=684) \text {, } \\
\text { proportion of patients, } \%\end{array}$ & OR $(95 \% \mathrm{Cl})$ & $p$-value \\
\hline \multicolumn{5}{|l|}{ 7-day PPA } \\
\hline Week 2 & 18.8 & 13.3 & $1.59(1.17-2.16)$ & 0.003 \\
\hline Week 13 & 34.1 & 23.7 & $1.72(1.35-2.20)$ & $<0.001$ \\
\hline Week 26 & 20.8 & 13.9 & $1.64(1.23-2.18)$ & 0.001 \\
\hline Week 26 (urine cotinine-confirmed) & 15.7 & 10.1 & $1.68(1.21-2.33)$ & 0.002 \\
\hline CAR weeks $26-52$ & 6.6 & 5.6 & $1.19(0.76-1.87)$ & 0.439 \\
\hline
\end{tabular}

$\mathrm{Cl}=$ confidence interval; $\mathrm{CAR}=$ complete abstinence rate; $\mathrm{OR}=$ odds ratio; $\mathrm{PPA}=$ point prevalence of abstinence.

were: abnormal dreams, 55 (8.1\%) and 17 (3.9\%); headache, 45 (6.6\%) and 23 (5.3\%); insomnia, 41 (6.0\%) and 25 (5.7\%); and nausea, 107 (15.7\%) and 49 (11.3\%).

\section{Discussion}

This is the first large-scale, pragmatic, adequately powered RCT conducted in Canadian ambulatory settings demonstrating that coverage of recommended pharmacotherapies for smoking cessation in motivated smokers significantly increases quit rates at weeks 2, 13, and 26. Using self-report and cotinine-confirmed abstinence measurements, respectively, the probability of quitting smoking at the end of the intervention in the full coverage group was 1.64 and 1.68 times larger than the probability of quitting in the no coverage group. Although there was a statistically significant difference between

Table 3 Reported and observed adverse events* (subjects randomized smoking and used smoking cessation medication) and Columbia Suicide Severity Rating Scale (C-SSRS) assessments ${ }^{\dagger}$

\begin{tabular}{lcc}
\hline All-causality AEs, $\boldsymbol{n}(\%)$ & $\begin{array}{c}\text { Full coverage } \\
(\boldsymbol{n}=\mathbf{6 8 2})\end{array}$ & $\begin{array}{c}\text { No coverage } \\
(\boldsymbol{n}=\mathbf{4 3 5})\end{array}$ \\
\hline Total number of AEs & 1473 & 830 \\
$\begin{array}{l}\text { Total number of participants } \\
\text { with AEs }\end{array}$ & $451(66.1)$ & $271(62.3)$ \\
$\begin{array}{l}\text { Subjects who discontinued } \\
\text { study due to AE }\end{array}$ & $6(0.9)$ & 0 \\
Subjects with SAEs & $35(5.1)$ & $18(4.1)$ \\
Subjects with severe AEs & $57(8.4)$ & $32(7.4)$ \\
\hline
\end{tabular}

$\mathrm{AE}=$ adverse event; $\mathrm{SAE}=$ serious adverse event.

*All observed or volunteered AEs, regardless of smoking cessation method, or selected or suspected causal relationship, were reported. For all AEs, the investigator was required to pursue and obtain information adequate both to determine the outcome of the $\mathrm{AE}$ and to assess whether it met the criteria for classification as a serious $\mathrm{AE}$.

${ }^{\dagger}$ The C-SSRS was part of the neuropsychiatric section of the medical history at screening/randomization and was administered to all subjects at given visits and at the investigator's discretion to assist with safety reporting and evaluation of possible suicide-related AEs. A "yes" answer for suicidal ideation and/or behavior was observed in 25 (3.7\%) and $11(2.5 \%)$ in the full coverage and no coverage groups, respectively (all-randomized and treated population). A suicidal attempt was reported in one subject who did not take smoking cessation medication. the self-reported and cotinine-confirmed quit rates, there was no significant difference between the groups (data not shown). The weeks 26-39 and weeks 26-52 CARs between the two groups did not differ significantly, possibly indicating the loss of this benefit upon withdrawal of pharmacotherapy coverage. Using self-reported 7-day PPA at week 26, for every 14.4 smokers who receive pharmacotherapy coverage, there will be one additional quitter. Of every 8.3 persons covered, one will experience an $\mathrm{AE}$ due to attempting to quit smoking, and of every 111 , one will discontinue due to a serious AE. Given that smoking kills one in two people, the risks of pharmacotherapy are lower than continuing to smoke.

The results of this study could be explained by greater medication adherence and utilization in the full coverage group, and a consequent greater number of quit attempts. In the no coverage group, the larger loss to follow-up may reflect real-life experience of smokers who receive cessation counselling, but do not complete their quit attempt because of lack of coverage. However, a higher than anticipated number of subjects (63.6\%) in the control group were dispensed pharmacotherapy during the intervention period. This could be explained by higher motivation of the smokers in this trial and the SmartPayment ${ }^{\mathrm{Tm}}$ card reimbursement of $\$ 5.00$ per pharmacy dispensation in the control group. Moreover, the study sites, although not based in academic centres, might not reflect other community ambulatory clinics. These clinics were selected to participate because of their interest in treating tobacco addiction and might have been more diligent in promoting medication to stop smoking, medication adherence, and restarting medications in those who failed to quit.

The results of our study are consistent with other studies [9,24,27]. However, our study had a greater number of quit attempts, use of pharmacotherapy, and slightly higher quit rates in both groups. This may be because our study enrolled motivated smokers and empowered the practitioner-patient dynamic by permitting tailoring of pharmacotherapy options during 
the coverage period. The loss of effectiveness of coverage by week 39 (13 weeks after coverage ceased) should be interpreted with caution because it is not clear if the diminished quit rates reflect the chronic relapsing nature of nicotine dependence, the erosion of the intervention effect over time [28], lack of statistical power, the intensity and frequency of the follow-up, or the stringency of the definition of a continuous quitter for a pragmatic, real-world trial. It should be noted that week-26 PPA was chosen as a pragmatic endpoint [11] and the study was not powered to detect statistical significance for CARs.

Despite the study limitations, several strengths should be highlighted. The study was community-based with minimal exclusion criteria; the majority of subjects were treated by their own physician; each smoker could choose their preferred quit method(s) (pharmacological and/or non-pharmacological); and subjects received personalized treatment rather than a standard protocol. Our study attempted to create a longer window than the usual 10-12 weeks of standard coverage during which participants could keep trying to quit and allowed for a switch to or combination with another medication. Subject recruitment was completed 5 months ahead of schedule, indicating the demand for services, easy adoption of the protocol, and feasibility.

\section{Conclusions}

The adoption of a pharmacotherapy coverage drug policy was shown to be an effective intervention to improve 26-week quit rates in Canada. Medication adherence needs to be promoted by prescribers to enhance quitting. Relapse prevention strategies that include preventing and managing slips through extended behavioural and or pharmacological interventions or combination medication might be required for smokers who respond to pharmacotherapy but relapse once medication is discontinued. Further research is needed to establish the actual duration of coverage required for sustained effects on smoking cessation.

\section{Additional files}

Additional file 1: Pre-screening, screening, and randomization procedures. Flow diagram illustrating the pre-screening, screening and randomization procedures involved in the ACCESSATION study.

Additional file 2: Dosage forms and strengths of smoking cessation pharmacotherapies eligible for reimbursement. Table showing the eligible pharmacological smoking cessation method(s) prescribed according to the most recent version of the respective product monograph or equivalent.

Additional file 3: Continuous abstinence rate [weeks 26-39 and 26-52]. Line graph showing continuous abstinence rates for after withdrawal of coverage eligibility (weeks 26 to 52) for those who had previously had full versus no coverage during the intervention phase (first 26 weeks of the study).
Additional file 4: Assumed smoking cessation pharmacotherapy utilization over $\mathbf{2 6}$ weeks. Plot showing pharmacotherapy utilization in the full versus no coverage groups during the intervention phase (first 26 weeks of the study) (post-hoc analysis).

\section{Competing interests}

Pfizer sponsored the study and recruited study sites based on various factors, including but not limited to, clinical research experience and capabilities, smoking cessation expertise/interest, and referral from peers. Data were analyzed by Pfizer and made available to the authors for interpretation and preparation of the manuscript. PS, GB, and PO did not receive honoraria for their participation or for writing the manuscript. VR, CA, and SR are employees of Pfizer Inc. PO, PS, and GB declare financial compensation from Pfizer Inc. for professional services, including protocol and clinical trial materials development, initial start-up, and end-of-study activities such as Case Report Form review, Statistical Analysis Plan review, preparation, participation, and presentation at the Investigator Meeting, and Clinical Study Report review. No payments were made by Pfizer Inc. to PO, PS, or GB for authorship and/or authorship-related activities of this paper. CA, SR, and VR are employees of and shareholders in Pfizer Inc.

\section{Authors' contributions}

$P S, G B, S R, P O$, and VR were involved in the design of the study. CA performed the statistical analysis of the data. PS, GB, SR, PO, CA, and VR interpreted the data. PS and GB wrote the first draft of the manuscript and made revisions. PS, $\mathrm{GB}, \mathrm{SR}, \mathrm{PO}, \mathrm{CA}$, and VR critically reviewed the manuscript for important intellectual content and accuracy. All authors have read and approved the final version to be published.

\section{Acknowledgements}

Dr. Jacques Le Lorier, MD, PhD; Professor, Department of Medicine \& Pharmacology, University of Montreal was involved in the elaboration of the initial concept, and contributed to the protocol design and discussion of the statistical analysis plan. The authors would like to thank Ching-Ray Yu, PhD, for his assistance with statistical analysis. This study was funded by Pfizer Inc. Editorial support was provided by Helen Jones, PhD and Anne Jakobsen, MSc of Engage Scientific and funded by Pfizer Inc.

\section{Author details}

${ }^{1}$ Addictions Division, Centre for Addiction and Mental Health, Toronto, Ontario, Canada. ${ }^{2}$ Departments of Family and Community Medicine and Psychiatry and the Dalla Lana School of Public Health, University of Toronto, Toronto, Ontario, Canada. ${ }^{3}$ Ontario Tobacco Research Unit, Toronto, Ontario, Canada. ${ }^{4}$ Department of Family Medicine, Dalhousie University, Halifax, Nova Scotia, Canada. ${ }^{5}$ Cardiac Rehabilitation and Secondary Prevention Program, Toronto Rehabilitation Institute, Toronto, Canada. ${ }^{6}$ Health Economics and Outcomes Research, Pfizer Canada Inc, Kirkland, Québec, Canada. ${ }^{7}$ Chantix/ Champix Project Statistical Lead, Pfizer Inc, New York, NY, USA. ${ }^{8}$ Pfizer Canada Inc, Kirkland, Québec, Canada.

Received: 4 October 2013 Accepted: 29 April 2014 Published: 7 May 2014

\section{References}

1. World Health Organization: WHO REPORT on the Global Tobacco Epidemic 2008. The MPOWER package. The MPOWER package [http://www.who.int/ tobacco/mpower/mpower_report_full_2008.pdf]

2. Stead LF, Perera R, Bullen C, Mant D, Lancaster T: Nicotine replacement therapy for smoking cessation. Cochrane Database Syst Rev 2008, 1, CD000146.

3. Hughes JR, Stead LF, Lancaster T: Antidepressants for smoking cessation. Cochrane Database Syst Rev 2007, 1, CD000031.

4. Cahill K, Stead LF, Lancaster T: Nicotine receptor partial agonists for smoking cessation. Cochrane Database Syst Rev 2012, 4, CD006103.

5. Tran K, Asakawa K, Cimon K, Moulton K, Kaunelis D, Pipe A, Selby P: Pharmacologicbased strategies for Smoking Cessation: Clinical and Cost-effectiveness Analyses. [http://wnw.ncbi.nlm.nih.gov/pmc/articles/PMC3442619/]

6. Eisenberg MJ, Filion KB, Yavin D, Bélisle P, Mottillo S, Joseph L, Gervais A, O'Loughlin J, Paradis G, Rinfret S, Pilote L: Pharmacotherapies for smoking 
cessation: a meta-analysis of randomized controlled trials. CMAJ 2008, 179(2):135-144.

7. Le Foll B, George TP: Treatment of tobacco dependence: integrating recent progress into practice. CMAJ 2007, 177(11):1373-1380.

8. Penz ED, Manns BJ, Hébert PC, Stanbrook MB: Governments, pay for smoking cessation. CMAJ 2010, 182(18):E810.

9. Reda AA, Kotz D, Evers SM, van Schayck CP: Healthcare financing systems for increasing the use of tobacco dependence treatment. Cochrane Database Syst Rev 2012, 6, CD004305.

10. Le Strat $Y$, Rehm J, Le Foll B: How generalisable to community samples are clinical trial results for treatment of nicotine dependence: a comparison of common eligibility criteria with respondents of a large representative general population survey. Tob Control 2011, 20(5):338-343.

11. Selby P, Brosky G, Oh P, Raymond V, Ranger S: How pragmatic or explanatory is the randomized, controlled trial? The application and enhancement of the PRECIS tool to the evaluation of a smoking cessation trial. BMC Med Res Methodol 2012, 12:101.

12. Thorpe KE, Zwarenstein M, Oxman AD, Treweek S, Furberg CD, Altman DG, Tunis S, Bergel E, Harvey I, Magid DJ, Chalkidou K: A pragmatic-explanatory continuum indicator summary (PRECIS): a tool to help trial designers. CMAJ 2009, 180(10):E47-E57.

13. Fagerstrom KO, Heatherton TF, Kozlowski LT: Nicotine addiction and its assessment. Ear Nose Throat J 1990, 69(11):763-765.

14. Heatherton TF, Kozlowski LT, Frecker RC, Fagerström KO: The Fagerström Test for Nicotine Dependence: a revision of the Fagerström Tolerance Questionnaire. Br J Addict 1991, 86(9):1119-1127.

15. Posner K, Oquendo MA, Gould M, Stanley B, Davies M: Columbia Classification Algorithm of Suicide Assessment (C-CASA): classification of suicidal events in the FDA's pediatric suicidal risk analysis of antidepressants. A J Psychiatry 2007, 164(7):1035-1043.

16. Fiore FC, Jaén CR, Baker TB, Bailey WC, Benowitz NL, Curry SJ: Treating Tobacco Use and Dependence: 2008 Update. Clinical Practice Guideline. Rockville, MD: U.S. Department of Health and Human Services Public Health Service; 2008.

17. Hughes JR, Keely JP, Niaura RS, Ossip-Klein DJ, Richmond RL, Swan GE: Measures of abstinence in clinical trials: issues and recommendations. Nicotine Tob Res 2003, 5(1):13-25.

18. Wong SL, Shields M, Leatherdale S, Malaison E, Hammond D: Assessment of validity of self-reported smoking status. Health Rep 2012, 23(1):47-53.

19. Gonzales D, Rennard SI, Nides M, Oncken C, Azoulay S, Billing CB, Watsky EJ, Gong J, Williams KE, Reeves KR: Varenicline, an alpha4beta2 nicotinic acetylcholine receptor partial agonist, vs sustained-release bupropion and placebo for smoking cessation: a randomized controlled trial. JAMA 2006, 296(1):47-55.

20. Jorenby DE, Hays JT, Rigotti NA, Azoulay S, Watsky EJ, Williams KE, Billing CB, Gong J, Reeves KR: Efficacy of varenicline, an alpha4beta2 nicotinic acetylcholine receptor partial agonist, vs placebo or sustained-release bupropion for smoking cessation: a randomized controlled trial. JAMA 2006, 296(1):56-63.

21. Kaper J, Wagena EJ, Severens JL, Van Schayck CP: Healthcare financing systems for increasing the use of tobacco dependence treatment. Cochrane Database Syst Rev 2005, 1, CD004305.

22. Kaper J, Wagena EJ, Willemsen MC, van Schayck CP: A randomized controlled trial to assess the effects of reimbursing the costs of smoking cessation therapy on sustained abstinence. Addiction 2006, 101(11):1656-1661.

23. Hughes JR, Keely J, Naud S: Shape of the relapse curve and long-term abstinence among untreated smokers. Addiction 2004, 99(1):29-38.

24. Kaper J, Wagena EJ, Willemsen MC, van Schayck CP: Reimbursement for smoking cessation treatment may double the abstinence rate: results of a randomized trial. Addiction 2005, 100(7):1012-1020.

25. West R: The clinical significance of "small" effects of smoking cessation treatments. Addiction 2007, 102(4):506-509.
26. Zwarenstein M, Treweek S, Gagnier JJ, Altman DG, Tunis S, Haynes B, Oxman AD, Moher D: Improving the reporting of pragmatic trials: an extension of the CONSORT statement. BMJ 2008, 337:a2390.

27. Schauffler HH, McMenamin S, Olson K, Boyce-Smith G, Rideout JA, Kamil J: Variations in treatment benefits influence smoking cessation: results of a randomised controlled trial. Tob Control 2001, 10(2):175-180.

28. Baker TB, Mermelstein R, Collins LM, Piper ME, Jorenby DE, Smith SS, Christiansen BA, Schlam TR, Cook JW, Fiore MC: New methods for tobacco dependence treatment research. Ann Behav Med 2011, 41(2):192-207.

doi:10.1186/1471-2458-14-433

Cite this article as: Selby et al:: A pragmatic, randomized, controlled study evaluating the impact of access to smoking cessation pharmacotherapy coverage on the proportion of successful quitters in a Canadian population of smokers motivated to quit (ACCESSATION). BMC Public Health 2014 14:433.

\section{Submit your next manuscript to BioMed Central and take full advantage of:}

- Convenient online submission

- Thorough peer review

- No space constraints or color figure charges

- Immediate publication on acceptance

- Inclusion in PubMed, CAS, Scopus and Google Scholar

- Research which is freely available for redistribution

Submit your manuscript at www.biomedcentral.com/submit
C) BioMed Central 